\title{
G protein-gated potassium channels: a link to drug addiction
}

Authors: Robert A. Rifkin ${ }^{1,2}$, Stephen J. Moss ${ }^{3,4}$ and Paul A. Slesinger ${ }^{1, *}$

\section{Author Affiliations:}

${ }^{1}$ Fishberg Department of Neuroscience and Friedman Brain Institute, Icahn School of Medicine at Mount Sinai, One Gustave L. Levy Place, New York, NY 10029

${ }^{2}$ Medical Scientist Training Program, Icahn School of Medicine at Mount Sinai

${ }^{3}$ Dept of Neuroscience, Tufts University School of Medicine, Boston, MA 02155

${ }^{4}$ Dept of Neuroscience, Physiology and Pharmacology, University College, London WC1E, 6BT

${ }^{*}$ Corresponding author: Paul A. Slesinger, Dept. of Neuroscience, Friedman Brain Institute, Icahn School of Medicine at Mount Sinai, One Gustave L. Levy Place, New York, NY 10029.

Email: paul.slesinger@mssm.edu 
Abstract: $G$ protein-gated inwardly rectifying potassium (GIRK) channels are regulators of neuronal excitability in the brain. Knockout mice lacking GIRK channels display altered behavioral responses to multiple addictive drugs, implicating GIRK channels in addictive behaviors. Here, we review the effects of GIRK subunit deletions on the behavioral response to psychostimulants, such as cocaine and methamphetamine. Additionally, exposure of mice to psychostimulants produces alterations in surface expression of GIRK channels in multiple types of neurons within the brain's reward system. We compare the subcellular mechanisms by which drug exposure appears to alter GIRK expression in multiple cell types and provide an outlook on future studies examining the role of GIRK channels in addiction. A greater understanding of how GIRK channels are regulated by addictive drugs may enable the development of therapies to prevent or treat drug abuse. 


\section{Glossary (450 words)}

- Baclofen: a selective high-affinity agonist for the $G A B A_{B}$ receptor.

- Conventional VTA DA neurons: In-positive, slower-firing VTA DA and SNc DA neurons that project to NAc lateral shell and striatum.

- $\mathbf{D}_{2}$ receptor: the dopamine receptor subtype 2, which couples to $\mathrm{G}_{\mathrm{i} / 0}$-type $\mathrm{G}$ proteins, and is an autoreceptor on midbrain DA neurons.

- DA: dopamine, a catecholamine neurotransmitter produced by cells in a small number of brain regions including VTA, SNc, hypothalamus and olfactory bulb.

- EPSC: Excitatory post-synaptic current. A depolarizing current evoked by a synaptically released excitatory neurotransmitter, such as glutamate.

- GABA: $y$-amino butyric acid. A primary inhibitory neurotransmitter in the brain that is an agonist for ionotropic $G A B A_{A}$ receptors and metabotropic $G A B A_{B}$ receptors.

- $\mathrm{GABA}_{B}$ receptor: An obligate heterodimer of $\mathrm{GABA}_{B 1 a b}$ and $G A B A_{B 2}$ receptor subunits that couples to $\mathrm{G}_{\mathrm{i} / \mathrm{o}} \mathrm{G}$ proteins.

- GIRK channel: a G protein-gated inwardly rectifying potassium channel.

- GPCR: G protein-coupled receptor. A seven transmembrane domain protein that couples to heterotrimeric $G$ proteins consisting of $\alpha, \beta$ and $y$ subunits. The $\alpha$ subunits are functionally divided into $\alpha_{i / 0}, \alpha_{s}, \alpha_{q / 11}$ and $\alpha_{12 / 13}$ families.

- $I_{h}$ current: an inward cationic current mediated by hyperpolarization-activated, cyclic nucleotide-gated $(\mathrm{HCN})$ channels. Activation of $\mathrm{I}_{\mathrm{h}}$ depolarizes neurons, contributing to "pacemaker" neuronal activity.

- LHb: Lateral habenula, a region of the diencephalon dorsal to the thalamus, which is activated by aversive stimuli, such as from cocaine withdrawal and foot shock.

- LC: Locus coeruleus, a nucleus in the pons, which is the main source of norepinephrine in the brain.

- MOR: $\mu$-opioid receptor. An opioid GPCR that couples to $\mathrm{G}_{\mathrm{i} / \mathrm{o}} \mathrm{G}$ proteins and is expressed on GABA neurons of the VTA and RMTg and in LC neurons.

- MSNs: medium spiny neurons. GABAergic neurons expressed throughout the striatum.

- MPFC: medial prefrontal cortex. A region of cerebral cortex associated with inhibition of unwanted behaviors and with executive function; hypoactivity is associated with addictive drug use and impulsive behavior.

- NAc: Nucleus accumbens. A ventral striatal structure associated with reward-based learning and addiction. 
- PDZ domain: Postsynaptic density-95, Discs large, ZZona occludens domain. A protein domain that binds PDZ motifs, such as those found in GIRK2C and GIRK3 subunits.

- PX domain: Phox homology domain. A protein domain found in sorting nexin (SNX) proteins that binds specific phospholipids, often localizing the sorting nexin to specific intracellular membrane locations.

- RMTg: Rostromedial tegmental nucleus, or tail of the VTA (tVTA). Contains GABA neurons that exert a tonic inhibitory effect on VTA DA neurons.

- slow IPSC: slow, inhibitory post-synaptic current. A metabotropic, inhibitory current evoked by a synaptically released inhibitory neurotransmitter, such as GABA or DA.

- SNc: Substantia nigra pars compacta. A midbrain DA nucleus lateral to the VTA that projects largely to dorsal striatum. SNc DA neurons are implicated in initiation of behaviors and Parkinson disease.

- Unconventional DA neurons: $\mathrm{I}_{h}$-negative, faster-firing VTA DA neurons that project to the mPFC, NAc core, NAc medial shell, and amygdala.

- VTA: Ventral Tegmental Area. A midbrain DA nucleus that project to targets including mPFC, NAc and amygdala. VTA DA neurons are implicated in reward/motivation and addiction. 


\section{Box 1: The mesocorticolimbic dopamine system and psychostimulants}

The mesocorticolimbic dopamine (DA) system consists of dopaminergic cell bodies located in the ventral tegmental area (VTA) of the midbrain ("meso") that project to many brain regions including medial prefrontal cortex (mPFC) ("cortico"), nucleus accumbens (NAc) ("limbic") and amygdala ("limbic"). VTA DA neurons are implicated in learning, reward and addiction [1, 2]. The VTA is composed of predominantly DA neurons $[3,4]$, of which a fraction co-release glutamate [5]. The VTA also contains $\sim 35 \%$ GABAergic and $\sim 2-5 \%$ pure glutamatergic neurons $[4,6]$. GABA interneurons in the VTA [3, 7], as well as in the adjacent rostromedial tegmental nucleus (RMTg) or "tail" of the VTA [8], exert an inhibitory effect on VTA DA neuron firing. The VTA is adjacent to the brain's other major DA nucleus, the substantia nigra pars compacta $(\mathrm{SNc})$, which projects primarily to the dorsal striatum and is implicated in motor behaviors and Parkinson disease. In addition to releasing DA from axon terminals, DA neurons also make dendrodendritic synaptic connections with other DA neurons [9] and release DA from the somatodendritic compartment into the VTA [10].

Recent studies [11,12] have demonstrated subpopulations of VTA DA neurons that project to different brain targets and exhibit unique electrophysiological properties (for a review, see [13]). The "conventional" DA neurons have a prominent, hyperpolarization-activated current $\left(\mathrm{Ih}^{-}\right.$ positive), slow spontaneous firing rate and project primarily to the NAc lateral shell. Another population of DA neurons, so-called "unconventional" DA neurons, lack the $\mathrm{I}_{\mathrm{h}}$ current $\left(\mathrm{I}_{\mathrm{h}}\right.$ negative) and have a faster spontaneous firing rate. These unconventional DA neurons project primarily to the mPFC, NAc core, NAc medial shell, and amygdala [11]. Because unconventional DA neurons lack the $\ln _{\mathrm{h}}$ current, they have been typically excluded from earlier experiments due to the exclusive use of electrophysiological criteria to identify DA neurons. Today, using retrograde labeling of DA neuron projections [11, 12] and Cre-lox dependent viral constructs [2], the unconventional DA neurons are now more easily studied. Notably, unconventional DA neurons projecting to mPFC lack significant amounts of GIRK2 protein and $D_{2} R$-activated GIRK currents [11]. In addition to receiving projections from the VTA, many of these target regions send reciprocal projections to VTA.

\section{Box 2: SNX27 control of GIRK channel trafficking}

Sorting Nexin 27 (SNX27) is a unique adaptor protein that binds and regulates a subset of GIRK channels via a specific PDZ binding motif [14], thus providing an additional layer of GIRK 
channel regulation that may contribute to changes in GIRK channel expression caused by neuronal activity or addictive drugs. SNX27 contains three functional domains: a PDZ domain [15]; a Phox Homology (PX) domain [15]; and a 4.1/ezrin/radixin/moesin (FERM)-like domain. The PDZ domain is highly specific for certain class I PDZ motifs, including those present in GIRK2C and GIRK3 subunits [14, 16]. The PDZ domain of SNX27 also binds and regulates other surface proteins [17-24]. The PX domain of SNX27 selectively binds phosphatidylinositol3-phosphate (PI3P), which is enriched in early endosomes (EE). Thus, SNX27 is targeted to early endosomes [14, 24]. The FERM-like domain of SNX27 contains a Ras-association (RA) domain, and is necessary for SNX27 regulation of GIRK channels [25], as well as other SNX27regulated proteins [26].

The effects of SNX27 on GIRK function are complex. Coexpression of SNX27 with GIRK channels in HEK293 cells [14] or in cultured hippocampal neurons [16] reduces receptoractivated GIRK currents. Further studies in HEK293 cells suggested that SNX27 exerts a negative regulatory effect on GIRK3-containing channels, perhaps due to the lysosomal targeting motif in GIRK3 [27]. Interestingly, ablation of endogenously expressed SNX27 in DA neurons in vivo also leads to significantly reduced $G A B A_{B} R-G I R K$ currents [28], suggesting that SNX27 may be involved in forward trafficking of GIRK channels to the plasma membrane. This effect of SNX27 is consistent with previous reports for SNX27 regulation of the $\beta 2$ adrenergic receptor [19] and other signaling proteins [29]. SNX27 is postulated to function as an adapter between the retromer complex and cargoes containing a class I PDZ motif [19]. The fact that a similar functional outcome, i.e., reduced GIRK currents, is achieved both by ectopic expression of SNX27 in vitro and ablation of SNX27 in vivo could indicate cell-type specific differences. Alternately, the relationship between the levels of SNX27 protein and GIRK channels expressed on the plasma membrane may follow an inverted U-shaped curve, where too little or too much SNX27 protein may reduce GIRK channel trafficking to the plasma membrane. This inverted Ushaped model is compatible with the hypothesis that SNX27 functions as an adapter between GIRK channels and the retromer complex. Such an adapter would require an appropriate stoichiometric relationship with retromer in order to avoid lysosomal degradation. It remains to be shown, however, whether increases in SNX27 expression that occur under physiological conditions can reduce GIRK surface expression in vivo.

\section{Main Text:}

\section{A role for GIRK channels in the response to addictive drugs}


G protein-gated inwardly-rectifying potassium ( $\mathrm{GIRK}$ or $\mathrm{K}_{\mathrm{IR}} 3$ ) channels are regulators of neuronal firing and are expressed in several key regions of the brain's reward system, e.g., the ventral tegmental area (VTA) and medial prefrontal cortex (mPFC) (Box 1). In this review, we discuss the current understanding of how addictive drugs, especially psychostimulants, alter the expression and function of GIRK channels, and conversely, how changes in GIRK channel expression regulate the behavioral response to psychostimulants. Recent experiments have begun to unravel the mechanisms by which addictive drugs alter GIRK channel signaling, but significant unanswered questions remain.

\section{Fundamental Properties of GIRK Channels}

GIRK channels are members of the large super-family of inwardly rectifying $K$ channels $\left(K_{\mathbb{R}}\right)$, in which the inward current is significantly greater than the outward current (Figure 1a). This property of rectification is caused by occlusion of the pore by intracellular $\mathrm{Mg}^{2+}$ [30] and polyamines [31]. At voltages near the resting membrane potential, the small outward $\mathrm{K}^{+}$current through GIRK channels hyperpolarizes the neuron, reducing membrane excitability. In contrast, at positive membrane potentials voltage-gated potassium channels $\left(K_{v}\right)$ are activated and conduct a larger outward $\mathrm{K}^{+}$current, repolarizing the neuron. Stimulation of $\mathrm{G}$ protein-coupled receptors (GPCRs) that couple to $G_{i / 0} G$ proteins, such as the $G_{A B A_{B}}$ [32], dopamine $D_{2}[33], \mu$ opioid [34], serotonin 5-HT ${ }_{1 A}$ [35], adenosine $A_{1}$ [36], acetylcholine $M_{2}$ [37], and adrenergic $\alpha_{2}$ [38] receptors, activates GIRK channels via the G protein $G \beta Y$ subunits [39-41]. Synaptic activation of GIRK channels via these metabotropic receptors occurs over a time course of seconds, which is slower than activation of fast, ionotropic receptors. This slow inhibitory postsynaptic current (IPSC) therefore provides a more sustained form of inhibition of neuronal activity.

Functional GIRK channels exist primarily as heterotetramers of GIRK subunits (GIRK1-4), each expressed via its own gene (Figure 1b) [42-45]. GIRK2 has three splice variants, GIRK2a, GIRK2b, GIRK2c, that are expressed in brain [46-48]. GIRK2 can uniquely form functional homotetramers [27, 47, 49].

Each GIRK subunit varies in its cytoplasmic N- and C-terminal domains, such that GIRK channels composed of different GIRK subunits are trafficked to different intracellular locations [27] (Figure 1c; for a review, see [50]). GIRK2 subunits contain N-terminal (DQDVESPV) and C-terminal (ELETEEEE) endoplasmic reticulum (ER) export signals [27], which may underlie the 
unique ability of these subunits to form homotetrameric channels [27, 47, 49]. On the other hand, GIRK1 or GIRK3 subunits cannot exit the ER as homotetrameric channels [27], but instead form heterotetramers, i.e., GIRK1/2, GIRK2/3 or GIRK1/3, which traffic properly to the plasma membrane as functional channels. GIRK2 contains an N-terminal Val/Leu motif that promotes endocytosis [27] in a phosphorylation-dependent manner [51]. GIRK3 has a Cterminal lysosomal degradation signal (YWSI) that reduces surface expression of GIRK3containing channels [27]. Finally, GIRK2C and GIRK3 contain a C-terminal PDZ motif (ESKV) that is important for trafficking [14]. Thus, GIRK tetramers composed of different subunit combinations will traffic differently, leading to variations in expression on the plasma membrane [27].

GIRK1, GIRK2 and GIRK3 subunits are widely expressed in the rodent brain [52, 53], although notably absent from medium spiny neurons in the caudate-putamen (striatum) [53]. GIRK1GIRK2 heterotetramers are believed to be the predominant form of GIRK channels expressed in brain [54]. However, there are important regional differences in GIRK channel composition. In the VTA, DA neurons express only GIRK2C and GIRK3, whereas VTA GABA neurons express GIRK1, GIRK2C and GIRK3 subunits [55]. By contrast, DA neurons in the substantia nigra pars compacta (SNc) predominantly express GIRK2 homotetramers, comprised of GIRK2a and GIRK2c splice variants [56]. Interestingly, the affinity of GIRK2/3 heterotetrameric channels for $G \beta y$ subunits is less than that for GIRK1/3 heterotetramers [57, 58], increasing the $E_{50}$ for $\mathrm{GABA}_{B}$ receptors coupling to GIRK channels in VTA DA neurons $[55,59]$. In the mPFC, excitatory pyramidal neurons express GIRK1, GIRK2 and GIRK3 [60]. In rat, GIRK1, GIRK2 and GIRK3 are expressed in locus coeruleus, habenula and amygdala [52]. These differences in GIRK subunit expression in neurons likely contribute to additional regulatory mechanisms for GIRK channels in the brain's reward system.

\section{Effects of GIRK knockouts on addictive behaviors}

Most addictive drugs, despite their structural diversity, converge on a final common pathway of elevating DA concentration in target regions, e.g., nucleus accumbens (NAc) and mPFC, of the mesocorticolimbic DA system (Box 1; for a review, see [61]). For example, cocaine inhibits the dopamine transporter (DAT) and thus blocks reuptake of DA from the synaptic cleft by VTA DA neuron terminals in the NAc, increasing the DA concentration synaptically and extrasynaptically [62]. Similarly, amphetamine and methamphetamine enter presynaptic terminals and dendritic compartments of DA neurons where they induce reverse transport of DA through the DAT [63]. 
In contrast, opioids are classically believed to inhibit GABA neurons in the RMTg and VTA, resulting in disinhibition of the DA neurons and increased DA release [7,64]. Addictive drugs also induce somatodendritic DA release and elevate the concentration of DA within the SNc/VTA [65].

Studies in mice lacking GIRK channel subunits suggest that GIRK channels play a role in regulating the behavioral response to addictive drugs including psychostimulants. Many of these experiments have been performed in whole-animal knockout mice, potentially complicating their interpretation. GIRK channels are expressed in many different types of neurons, including those within the mesocorticolimbic DA system that have opposing roles, e.g., excitatory and inhibitory neurons. Thus, the effects observed in these studies represent the net effect of GIRK channels at multiple nodes within the system. Recently, experiments using cell-type specific knockout mice have provided more granular information.

\section{Whole-Animal Knockout Experiments}

An early study investigating GIRK2 ${ }^{-/-}$and $\mathrm{GIRK}^{-/-}$knockout mice in locomotor sensitization with psychostimulants exemplifies the complex nature of interpreting results from whole-animal knockouts [66] (see Table 1, Key Table). To induce locomotor sensitization, mice are injected daily with a psychostimulant over multiple days and the total distance moved is measured during the testing period. By the fifth day of injections, the locomotor response is significantly larger than on the first day of injection, i.e., sensitized. GIRK2 ${ }^{-/}$knockout mice exhibit an enhanced locomotor sensitization response to cocaine injections (15 mg/kg; i.p.) on days 4 and 5, compared to locomotor sensitization of littermate controls [66]. The locomotor sensitization response with cocaine in GIRK3 ${ }^{-/}$knockout mice, on the other hand, is indistinguishable from that of wild-type mice. The double knockout GIRK2 ${ }^{-1} / \mathrm{GIRK}^{--}$mice, however, have a cocaine sensitivity similar to WT [66]. In addition, GIRK2 ${ }^{--}$and GIRK3 ${ }^{-/-}$knockout mice both self-

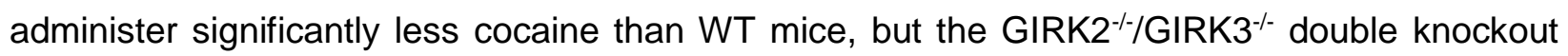
mice exhibit a cocaine sensitivity intermediate between the single knockouts and WT [66]. More recent experiments demonstrated that $\mathrm{GIRK} 1^{-/}$and $\mathrm{GIRK} 2^{-/-}$mice exhibit similarly enhanced behavioral responses to cocaine [67], yet VTA DA neurons lack GIRK1 [55] and have normal GIRK currents [67]. These findings suggest that changes in behavior in whole-animal GIRK channel knockouts can be driven by increases in VTA DA neuron intrinsic excitability but can also be affected by changes elsewhere in the brain. Indeed, changes in excitatory synaptic transmission have been reported for GIRK2 ${ }^{-/}$knockout mice [67]. Overall, these studies 
implicate all three GIRK subunits in regulating the behavioral response to cocaine but illustrate that these subunits interact in a complex manner in different regions of the brain.

Opioids signal through $\mu$ opioid receptors (MOR) which couple to GIRK channels in VTA and RMTg GABA neurons [3, 64] and in locus coeruleus (LC) neurons [68]. Classically, it has been believed that activation of MOR on VTA and RMTg GABA neurons activates GIRK channels, inhibiting these neurons and thus removing their tonic inhibition of VTA DA neurons, i.e., disinhibition [7, 61, 64]. Accordingly, deletion of GIRK channels in GABA neurons would be expected to prevent inhibition of GABA neurons, increasing their firing rate and the resultant inhibition of DA neurons, reducing the rewarding effects of morphine. Paradoxically, wholeanimal GIRK1 ${ }^{-1}$ and GIRK2 ${ }^{-1}$ knockout mice exhibit an increased locomotor response to morphine, while GIRK3 ${ }^{-1}$ KO mice are significantly less responsive to morphine-induced locomotor activity [69] (for additional details, see the discussion of cell type-specific knockouts below). Expression of the GIRK3 subunit in only the VTA of GIRK3 ${ }^{-1}$ knockout mice restores morphine-induced motor activity, specifically implicating the GIRK3 subunit in the VTA for this behavior [69]. Both GIRK1 and GIRK2, however, appear to contribute to the analgesic effects of morphine [70]. In LC neurons, the increase in firing associated with withdrawal is absent in whole-animal GIRK2 $2^{-1 / G_{R K}}{ }^{-1} \mathrm{KO}$ mice, suggesting that morphine withdrawal symptoms, but not analgesia, are attenuated due to changes in the LC [68].

Whole-animal GIRK knockout mice also demonstrate altered effects with other addictive substances, such as ethanol. GIRK2 $2^{-/}$knockout mice display increased ethanol consumption, increased open-field motor activity with ethanol, reduced anxiolytic effects of ethanol, and reduced severity of withdrawal [71]. Whole-animal $\mathrm{GIRK}^{-/}$and $\mathrm{GIRK}^{+/-}$mice exhibit conditioned place preference for ethanol (i.p. injections) under conditions that do not induce place preference in WT littermates [72]. On the other hand, whole-animal GIRK3 ${ }^{-1 /}$ mice exhibit less severe ethanol withdrawal [73] and an increase in ethanol binge drinking [74], suggesting that the expression of GIRK3 reduces ethanol drinking. In this latter study, viral expression of GIRK3 in the VTA restores the normal alcohol drinking in the GIRK3 ${ }^{--}$mice, providing more direct evidence that GIRK3 in the VTA is important for these ethanol-dependent behaviors [74].

Thus, whole-animal knockout studies strongly implicate GIRK channels in the response to multiple addictive drugs. However, GIRK channels are widely expressed and can induce adaptations elsewhere in a complex system, making it challenging to draw conclusions about the role of GIRK channels in specific cell types in mediating these behaviors. Cell type-specific rescues in the context of the whole-animal knockout may provide some clarity by narrowing the 
region(s) involved in the behavioral response, e.g., expression of GIRK3 in a specific target region of the whole-animal GIRK3 ${ }^{-/} \mathrm{KO}$ mice.

\section{Cell-type Specific Knockout Experiments}

Recent cell-type specific knockout experiments have begun to shed new light on the role of GIRK channel subunits in specific cell types in addiction. In these experiments, a mouse line is generated in which a Girk subunit exon is flanked by loxP sequences (termed "floxed"), e.g., Girk $2^{\mid t / f l}$. The Girk $2^{|/| f l}$ line is then crossed with a second mouse line carrying a gene for Cre recombinase under the control of a cell-type specific promoter, e.g., the promoter for tyrosine hydroxylase (TH-Cre). The floxed gene is permanently excised in only cells expressing Cre recombinase. For studying the function of GIRK channels in the reward pathway, Cre lines are available for targeting DA neurons, using the promoter for DA transporter (DAT-Cre) or TH-Cre (but see [75] for additional discussion on these Cre lines); and for GABA neurons, using the promoter for glutamic acid decarboxylase (GAD2-Cre).

MOR-dependent activation of GIRK channels in VTA and RMTg GABA neurons is hypothesized to enhance DA neuron firing via disinhibition [7, 61, 64]. However, cell-type specific deletion of GIRK2 subunits from GABA neurons (GAD2-Cre ${ }^{+/-}:$Girk2 $\left.2^{\mid t / f l}\right)$, which reduces GIRK currents in GABA but not DA neurons of VTA, has no effect on morphine-induced locomotor behavior [69]. Conversely, specific deletion of GIRK2 from DA neurons (DAT-Cre ${ }^{+/-}:$Girk2 $2^{i / f l}$ mice) elevates the locomotor response to morphine $(10 \mathrm{mg} / \mathrm{kg})$ [69]. These data suggest that, contrary to prediction, the motor response to morphine is not dependent on GIRK signaling in GABA neurons but is instead influenced by the modulatory effect of GIRK signaling in VTA DA neurons. A MOR-mediated presynaptic inhibition in GABA neurons may also remain in these knockout animals, leaving open the possibility of a disinhibition of VTA DA neurons as an additional mechanism of opioid action.

Previous studies with whole-animal knockout of GIRK2 have indicated increased locomotor response to cocaine [66]. Studies with a cell-type specific ablation of GIRK2 in DA neurons (DAT-Cre $^{+/}:$Girk2 $^{\mathrm{fl} / \mathrm{fl}}$ or GIRK2 $\mathrm{DA}$ KO mice) reveal enhanced acute and chronic locomotor response to cocaine [76]. In cocaine self-administration experiments, GIRK2DA KO mice exhibit an increased number of cocaine infusions, particularly at low doses, and an increase in total intake of cocaine [76]. Interestingly, conditioned place preference with cocaine is unaffected by GIRK2 knockout [76]. Overall, these conditional GIRK2 knockout experiments support the view 
that expression of GIRK channels in VTA DA neurons plays an important modulatory role in the behavioral responses to cocaine and morphine.

Another approach to studying the role of GIRK in addiction is to target a GIRK regulatory protein. Sorting nexin 27 (SNX27), originally identified as methamphetamine responsive transcript 1 (mrt1) [15, 77] binds to GIRK channels and regulates their trafficking [14] (Box 2). A cell-type specific knockout of SNX27 in DA neurons (SNX27DA KO) results in significantly attenuated $\mathrm{GABA}_{B} \mathrm{R}$-GIRK currents [28]. Additionally, the acute locomotor response produced by a single injection of cocaine $(20 \mathrm{mg} / \mathrm{kg})$, i.e., fold change relative to saline, is increased in SNX27 ${ }_{D A} K O$ mice relative to controls. The decrease in GABA $A_{B}$ R-GIRK current and the cocaineinduced locomotor effects are both rescued by viral expression of the SNX27-insensitive GIRK2a subunit, which lacks the PDZ motif, in VTA DA neurons [28]. These findings demonstrate that reducing GIRK surface expression in VTA DA neurons enhances the behavioral response to cocaine. It remains to be seen whether SNX27 plays a role in other addictive behaviors, such as locomotor sensitization, conditioned place preference or intravenous self-administration.

Together, these experiments highlight the benefit of studying cell-type specific knockout mice. More conditional knockouts with Girk $2^{|| / f l}$ or $S n \times 27^{|/| f \mid}$ mice and other Cre-lines, as well as developing new floxed lines for Girk $1^{\mathrm{fl} / \mathrm{fl}}$ and Girk $3^{\mathrm{fl} / \mathrm{fl}}$, are needed to better understand the roles of GIRK subunits in different neurons in the reward pathway. Additionally, Cre-dependent viral knockdown experiments [78] will enable dissection of changes in projection-specific pathways.

\section{Mechanisms of GIRK channel regulation in health and addiction}

Addictive drugs are believed to act opportunistically via cellular pathways that have evolved for other, adaptive purposes, such as synaptic plasticity (for a review, see [61]). Experiments in hippocampal and VTA neurons have uncovered some of the basic mechanisms regulating $\mathrm{GABA}_{B}$ receptor and GIRK channel expression. These mechanisms are similar to those mediating excitatory long-term potentiation (LTP) and inhibitory long-term depression (LTD), suggesting that excitatory glutamate and inhibitory $\mathrm{GABA}_{B} \mathrm{R}-\mathrm{GIRK}$ signaling are coordinately regulated to maintain excitatory-inhibitory balance. Additional studies have begun to elucidate the effect of psychostimulant exposure on GIRK channel expression and function in distinct cell types within the mesocorticolimbic system. Although psychostimulants have been most widely studied in the context of GIRK channel plasticity, it is possible that other addictive drugs may 
exert similar effects on GIRK channel function but have yet to be uncovered. Addictive drugs can hijack intrinsic mechanisms of neuronal plasticity, setting a course for becoming addicted.

\section{LTP of glutamatergic synapses concomitantly potentiates $G A B A_{B} R$-GIRK currents}

GIRK channels exert a profound effect on neuronal activity and have recently been shown to be subject to activity-dependent plasticity changes. For example, induction of long term potentiation (LTP) of the excitatory post-synaptic current (EPSC) leads to sustained potentiation of the $\mathrm{GABA}_{B} \mathrm{R}$-GIRK slow IPSC [79]. In these experiments, electrical stimulation of the border of stratum radiatum and stratum lacunosum-moleculare evokes a slow IPSC in rat CA1 pyramidal neurons that is carried by $\mathrm{GABA}_{B}$ receptors, i.e. SCH5091-sensitive, and GIRK channels [79]. An LTP pairing protocol consisting of $3 \mathrm{~Hz}$ stimulation paired with depolarization for 2.5 minutes induces LTP of the slow IPSC [79]. Like LTP of the EPSC, LTP of the slow IPSC depends on $\mathrm{Ca}^{2+}$ influx through the NMDA receptor and activation of CaMKII, indicating a similar mechanism for potentiating both excitatory and slow-inhibitory currents [79]. GABA $R$, GIRK1 and GIRK2 are expressed in the dendritic shaft as well as the dendritic spine, which is the site of LTP of the EPSC [79-81].

$\mathrm{GABA}_{B} \mathrm{R}$-GIRK currents in VTA DA neurons are also potentiated in response to increased neuronal activity. In VTA brain slice recordings, a CGP54626-sensitive slow IPSC, i.e., $\mathrm{GABA}_{B} \mathrm{R}$-GIRK current, is evoked by electrical stimulation (10 pulses at $66 \mathrm{~Hz}$ ) of predominantly GABA interneurons [82]. Induction of burst firing in VTA DA neurons (5 pulses at $20 \mathrm{~Hz}$, every 500 ms for 5 minutes) increases the amplitude of the GABA ${ }_{B}$ R-GIRK slow IPSC, whereas tonic firing (2 $\mathrm{Hz}$ for 2.5 minutes) decreases the GABA ${ }_{B} R-G I R K$ slow IPSC [82]. Burst firing also potentiates the $D_{2}$-GIRK current and GTP $\gamma S$-evoked current, suggesting that the increase is mediated by increased surface expression of GIRK channels. Like LTP in the hippocampus, the potentiation is dependent on $\mathrm{Ca}^{2+}$ influx through NMDA receptors and activation of CaMKII [82]. Interestingly, potentiation of the GABA ${ }_{B}$ R-GIRK currents via burst firing is absent in GIRK3 ${ }^{--}$ knockout mice. Furthermore, application of a dominant-negative peptide targeting the PDZ motif of GIRK2c or GIRK3 prevents the activity-dependent potentiation. Together, these experiments suggest that trafficking of GIRK channels via their PDZ motifs may be necessary for the plasticity to occur [82]. It remains to be determined if the PDZ domain-containing protein SNX27 is involved in this form of plasticity (Box 2). 
Thus, several protocols that induce excitatory glutamatergic activity also potentiate inhibitory GABA ${ }_{B}$ R-GIRK currents, inducing a form of homeostatic plasticity. The enhancement of $\mathrm{GABA}_{B} \mathrm{R}$-GIRK currents would be expected to partially counteract the effect of excitatory LTP on overall neuronal excitability, maintaining excitatory-inhibitory balance, which may be lost in disease states.

\section{NMDAR activation upregulates $A_{1} R$-GIRK currents but downregulates $G A B A_{B} R$-GIRK currents}

In dissociated hippocampal neurons, NMDA receptor activation increases surface expression of GIRK1 and GIRK2, via PP1 dephosphorylation of GIRK2 serine 9 (S9), which is located near a $\mathrm{Val} /$ Leu internalization motif [51]. Interestingly, NMDA receptor activation increases adenosine $A_{1}$ receptor-activated, but not GABA $A_{B}$ receptor-activated, GIRK current [83]. LTP of excitatory synapses induced by high frequency stimulation can be depotentiated, that is reversed, by low frequency stimulation (LFS). LFS potentiates $A_{1} R$-GIRK currents via the same PP1-dependent mechanism [83]. GIRK2 ${ }^{-/}$mice fail to exhibit depotentiation, indicating a requirement for GIRK2 and providing further support that changes in GIRK2 trafficking underlie the plasticity in synaptic signaling [83].

In addition to regulating GIRK channel expression, glutamatergic signaling through NMDA receptors can also modulate expression of the $\mathrm{GABA}_{B}$ receptor. Prolonged activation of NMDA receptors in neurons decreases surface expression of $G A B A_{B} R$ and reduces the amplitude of the $\mathrm{GABA}_{\mathrm{B}} \mathrm{R}$-GIRK current [84]. In this case, NMDA receptor activation, $\mathrm{Ca}^{2}$ influx, and an increase in CaMKII-dependent phosphorylation trigger endocytosis of $G_{A B A_{B}}$ receptors and reduction of the $G A B A_{B} R-G I R K$ current [84]. Phosphorylation of the $G A B A_{B 1}$ receptor subunit at serine 867 (S867) appears to be involved in $G_{A B A} R$ trafficking [84]. Similarly, sustained activation of NMDA receptors also reduces surface expression of $G A B A_{B} R$ via diversion from a recycling to degradation pathway [85]. This diversion of $G A B A_{B} R$ to lysosomal degradation is dependent on dephosphorylation of serine at 783 (S783) in $\mathrm{GABA}_{\mathrm{B} 2}$ receptor by a protein phosphatase, PP2A [85].

\section{$D_{2} R$-GIRK currents in midbrain DA neurons undergo activity- and DA-dependent LTD}

VTA and SNc DA neurons release DA from their somatodendritic compartments and induce a $\mathrm{D}_{2} \mathrm{R}$-GIRK slow IPSC in neighboring midbrain DA neurons [86]. Low-frequency stimulation or elevated DA induces LTD of the $D_{2} R$-GIRK slow IPSC, but not the GABA ${ }_{B} R-G I R K$ slow IPSC, in 
midbrain DA neurons [87]. Although dependent on intracellular calcium, the $D_{2} R-G I R K ~ L T D$ does not require calcium influx via NMDA or AMPA receptors (i.e. glutamatergic stimulation) but rather $D_{2} R$ desensitization [87]. The $D_{2} R$ has two isoforms, $D_{2}$-short $\left(D_{2 S}\right)$ and $D_{2}$-long $\left(D_{2 L}\right)$, and in mice expressing only a single isoform, calcium-dependent desensitization was shown to be mediated by the $D_{2 s}$ receptor [88].

Thus, GIRK channels couple to different GPCRs (e.g. GABA $A_{B}, A_{1} R, D_{2} R$ ) and these populations of GIRK channels may be differentially regulated. This could be accomplished, for example, if $A_{1}$ receptors and $G A B A_{B}$ receptors couple to different populations of GIRK channels, forming distinct pools of receptors and channels. In addition, distinct mechanisms of phosphorylation and de-phosphorylation of amino acids on GIRK channel subunits or on specific GPCRs provide another layer of differential regulation, which modulates neurotransmitter-evoked GIRK currents in the context of overall changes in excitability. These physiological plasticity mechanisms also appear to play a role in drug-induced changes in GIRK signaling in the VTA and mPFC.

\section{Effects of psychostimulants in non-DA neurons of the reward system}

Twenty-four hours after a single (i.p.) injection of methamphetamine $(2 \mathrm{mg} / \mathrm{kg}$ ) or cocaine (15 $\mathrm{mg} / \mathrm{kg}$ ), doses which are sufficient to induce locomotor sensitization [89], the amplitude of the $\mathrm{GABA}_{\mathrm{B}} \mathrm{R}$-depenent slow IPSC as well as the baclofen-evoked GABA $\mathrm{B}$-GIRK current (I Baclofen$_{\text {) }}$ are significantly smaller in VTA GABA neurons [90] (Figure 2). Notably, the suppression of $\mathrm{GABA}_{B} \mathrm{R}$-GIRK currents persists for at least one week [90], similar to the persistence in altered glutamatergic signaling on VTA DA neurons [91]. This $D_{1} R$ - and $D_{2} R$-mediated decrease in $\mathrm{GABA}_{\mathrm{B}} \mathrm{R}$-GIRK currents involves dephosphorylation of the $\mathrm{GABA}_{\mathrm{B} 2}$ subunit at serine 783 (S783) and subsequent internalization of the receptor and channel [90]. Acute inhibition of PP2A with okadaic acid, a phosphatase inhibitor, recovers the methamphetamine-depressed $\mathrm{I}_{\text {Baclofen }}$ [90], indicating that the changes in trafficking are highly dynamic. The persistence of this adaptation for 7 days suggests the exposure to psychostimulants is imprinted on the VTA circuitry. The subcellular mechanism underlying this persistent change is unknown, but could involve epigenetic changes [92] or selective changes in the distribution or activity of kinases and phosphatases. 
Decreased expression of inhibitory $G A B A_{B} R$-GIRK currents on GABA neurons is predicted to increase their firing rate, increasing the release of GABA onto VTA DA neurons and inhibiting VTA DA neuron firing. Thus, the downregulation of GABA ${ }_{B}$ R-GIRK currents in VTA GABA neurons in response to acute psychostimulants could represent an early, "protective" or homeostatic adaptation, which partially compensates for the increased VTA DA neuron excitability associated with drug exposure [91].

In the mPFC, a decrease in GABA ${ }_{B} R$-GIRK currents is also observed in layer $5 / 6$ glutamatergic pyramidal neurons of the prelimbic cortex (PrLC) following five days of cocaine (i.p.) injections [60] (Figure 2). As in VTA GABA neurons, cocaine exposure reduces phosphorylation of $\mathrm{GABA}_{\mathrm{B} 2} \mathrm{S783}$, and can persist for 28-40 days [60]. Acute application of okadaic acid also restores the cocaine-depressed GABABR-GIRK currents. Knockdown of GIRK1 and GIRK2 in mPFC leads to accelerated behavioral sensitization to cocaine [60]; thus, the downregulation of $\mathrm{GABA}_{B} \mathrm{R}$-GIRK currents in PrLC represents a maladaptive change that may support progression toward uncontrolled use.

The lateral habenula ( $\mathrm{LHb}$ ), a diencephalon structure located dorsal to the thalamus, is activated by aversive stimuli associated with cocaine withdrawal [93, 94]. Recently, an aversive stimulus, i.e., inescapable foot shock, was shown to decrease GABA R-GIRK currents in LHb neurons [95]. Though the stimulus was different, the plasticity in $G A B A_{B} R-G I R K$ currents may depend on the same PP2A-mediated GABA ${ }_{B 2}$ S783 dephosphorylation mechanism described in VTA GABA neurons with psychostimulants [90, 95]. To confirm this, additional experiments are needed to determine whether there is a change in phosphorylation of $G_{A B A} A_{B 2} S 783$. Significantly, systemic administration of a PP2A inhibitor, LB-100, ameliorated the depressivelike behavioral effects of inescapable foot shock [95].

Together, these studies highlight three different types of non-DA neurons within the brain's reward system that undergo a PP2A-dependent decrease in GABA $\mathrm{B}_{2} \mathrm{~S} 783$ phosphorylation and reduction in $G A B A_{B} R-G I R K$ currents following activation of the reward/aversion pathway. Because the effect of reward/aversion is mediated over different time courses and in different neurons within the reward circuitry, we expect the outcome to have divergent effects on behavior. The ability of systemic LB-100 treatment to reverse depressive behaviors mediated by downregulation of GABA ${ }_{B}$ R-GIRK currents [95] points to a promising avenue for the treatment of addiction. 


\section{Effects of psychostimulants on GIRK expression and regulation in VTA DA neurons}

In VTA DA neurons, acute cocaine or methamphetamine exposure reduces GABA $_{B} R-G I R K$ currents in a $D_{2}$-like $D A$ receptor dependent manner via a reduction in surface expression of GIRK channels [90, 96]. Similarly, chronic injections (i.p.) of methamphetamine (5 days) in a novel environment reduce GABA ${ }_{B}$ R-GIRK currents 24-48 hours after the last injection [97] (Figure 2). This decrease in GABA $A_{B} R$-GIRK current requires activation of both $D_{1}$ - and $D_{2}$-like DA receptors, and is likely determined by a reduction in GIRK channel surface expression because direct $G$ protein activation of GIRK channels using GTPYS is significantly reduced [97]. The methamphetamine-dependent decrease of $G A B A_{B} R-G I R K$ current persists in GABA $A_{B 2}-$ S783A mice, ruling out PP2A-dependent dephosphorylation of GABA $\mathrm{B}_{2} \mathrm{~S} 783$ as a possible mechanism [97]. Instead, expression of the GIRK3 subunit appears to be required for the methamphetamine-dependent decrease in GIRK current [97].

A single injection of cocaine (20 mg/kg; i.p.) is also sufficient to alter regulation of $D_{2} R-G I R K$ currents. As described above, normally this $D_{2} R$-GIRK slow IPSC undergoes calciumdependent desensitization [87], but following cocaine treatment, this calcium-dependent desensitization is eliminated [88]. Interestingly, although calcium-dependent desensitization is mediated via the $D_{2 S}$ receptor, only mice expressing both $D_{2 S}$ and $D_{2 L}$ receptors were altered by cocaine exposure [88], implying a role for both receptors in cocaine-dependent plasticity (Figure 2).

In addition to experimenter-mediated injections, mice can be trained to self-administer psychostimulants intravenously [98]. In a typical self-administration experiment, naïve mice are trained to lever press for an infusion of cocaine or methamphetamine, and the drug schedule or dose is then varied to assess the level of motivation for the drug [99]. In both VTA and SNc DA neurons, self-administration of methamphetamine reduces $D_{2} R$ - and GABA $R$-GIRK currents, confirming the effect of direct methamphetamine injections [100]. Interestingly, chelating intracellular $\mathrm{Ca}^{2+}$ with BAPTA promoted recovery of the $\mathrm{D}_{2} \mathrm{R}$ - and $\mathrm{GABA}_{\mathrm{B}} \mathrm{R}$-activated GIRK currents, suggesting a $\mathrm{Ca}^{2+}$-dependent mechanism of regulating function and/or trafficking (Figure 2). Future studies will need to determine whether this pathway overlaps with the GIRK3dependent mechanism implicated in non-contingent models of drug exposure [97].

In summary, psychostimulant exposure with both acute and chronic administrations decreases the amplitude of GABA $A_{B}$-GIRK currents in VTA DA neurons. Given that increases in VTA DA neuron excitability are associated with addictive behaviors, these reductions in GABA ${ }_{B} R-G I R K$ inhibition of excitability are likely maladaptive. In contrast to VTA GABA and mPFC pyramidal 
neurons, changes in GABA $A_{B} R-G I R K$ expression in VTA DA neurons in response to stimulants depend on the expression of the GIRK3 subunit [97]. Similarly, activity-dependent plasticity measured in brain slices is also dependent on the GIRK3 subunit [82]. Given that SNX27 binds GIRK2C and GIRK3 subunits via their PDZ motifs, and that SNX27 expression levels may be altered by psychostimulants $[15,77]$, it is possible that SNX27 may serve as a 'missing link' between psychostimulant exposure and changes in GIRK channel expression levels in VTA DA neurons (Box 2). Thus, the lack of GIRK1 expression in VTA DA neurons may provide a unique pathway for regulation of GIRK2C- and GIRK3-containing channels by SNX27 or other unidentified proteins.

\section{Concluding Remarks}

Significant advances have been made in defining a role for GIRK channels in the response to psychostimulants and other addictive drugs. GIRK channels regulate the behavioral response to addictive drugs, and conversely, exposure to addictive drugs alters the expression level of GIRK channels in the brain's reward circuitry. We suggest that future experiments should focus on several important areas as detailed below.

First, researchers should continue to use conditional knockouts and behavioral rescues in future experiments. To date, a majority of behavioral tests have used whole-animal knockouts. While very informative, the next level of analysis will need to focus on GIRK channel subtypes expressed in a particular type of neuron (Outstanding Question \# 1). For example, what is the role of the GIRK1 or GIRK3 subunit in VTA DA neurons? What is the role of the GIRK1 or GIRK3 subunit in mPFC pyramidal neurons? Conditional knockouts, using Cre-Lox methodology and cell-type specific promoters, will provide new tools for elucidating the function of GIRK channels in response to psychostimulants and other drugs.

Second, there should be an increase in studies investigating projection-specific pathways using retrograding viruses (Outstanding Question \# 2). As we learn more about the diversity of neuronal types within a brain region, it becomes essential to study the function of GIRKs within these subpopulations. For example, researchers have asked about the role of GIRK channels in conventional VTA DA neurons, but less is known about the unconventional VTA DA neurons. Using cell type-specific retrograde labeling techniques, e.g., Cre-dependent retrograde viral reporter constructs, it is possible to label, for example, VTA DA neurons targeting mPFC or NAc. 
Third, researchers should look at other brain regions outside the "classical" mesocorticolimbic DA pathway, such as lateral habenula and hippocampus, which also play a role in reward learning (Outstanding Question \# 3). Similar use of conditional knockouts and retrograde tracing will allow elucidation of the function of GIRK channels in specific cell types and populations within these non-dopaminergic brain regions.

Fourth, future studies should aim to identify upstream proteins that mediate the persistent changes in GIRK expression following drug exposure (Outstanding Question \# 4). We lack a detailed molecular understanding of how psychostimulants trigger changes in GIRK expression levels, especially in VTA DA neurons. Although the GABA ${ }_{B 2}$ S783 dephosphorylation mechanism can explain downregulation of $G A B A_{B} R-G I R K$ channels in non-DA neurons, it appears not to be responsible for the same effect observed in VTA DA neurons. Instead, GIRK3 subunit expression is somehow required for $G_{A B A_{B} R-G I R K}$ downregulation by psychostimulants. However, little else is known about this mechanism. Since SNX27 appears to regulate GIRK channels via the PDZ domains on GIRK2C and GIRK3, SNX27 is a good candidate for mediating some of the effects of psychostimulants.

Finally, it will be important to develop GIRK subunit-specific small-molecule drugs (Outstanding Question \# 5). Understanding the roles of distinct GIRK subunits, and potentially therapeutically targeting those subunits, would greatly enhance the development of subunit-specific small molecule agonists or antagonists. For example, ML297 is a selective activator of GIRK1containing GIRK channels [101], and has been shown to have antiepileptic properties [102]. However, a drug activating GIRK2 or GIRK3 subunits does not currently exist. A small molecule activator of GIRK3-containing channels would be particularly useful given the potentially important role played by this subunit in VTA DA neurons.

Acknowledgements: We thank members of the Slesinger lab for reading the manuscript. Work cited in this review was supported by grants from the NIDA (DA037170 to PAS \& SJM, \& F30DA039637 to RAR), NINDS (NS051195, NS081986 \& NS087662 to SJM), NIMH (MH097446, MH106954 to SJM), NIAAA (AA018734 to PAS) and DOD AR140209 (SJM).

Conflict of Interest: RAR and PAS have no conflicts of interest to report. SJM also serves as a consultant for Alcobra Therapeutics, AstraZeneca, and SAGE Therapeutics relationships that are regulated by Tufts University. 


\section{Citations}

1. Di Chiara, G. and A. Imperato, Drugs abused by humans preferentially increase synaptic dopamine concentrations in the mesolimbic system of freely moving rats. Proc Natl Acad Sci U S A, 1988. 85(14): p. 5274-8.

2. Tsai, H.C., et al., Phasic firing in dopaminergic neurons is sufficient for behavioral conditioning. Science, 2009. 324(5930): p. 1080-4.

3. Johnson, S.W. and R.A. North, Two types of neurone in the rat ventral tegmental area and their synaptic inputs. J Physiol, 1992. 450: p. 455-68.

4. Nair-Roberts, R.G., et al., Stereological estimates of dopaminergic, GABAergic and glutamatergic neurons in the ventral tegmental area, substantia nigra and retrorubral field in the rat. Neuroscience, 2008. 152(4): p. 1024-31.

5. Yamaguchi, T., et al., Mesocorticolimbic glutamatergic pathway. J Neurosci, 2011. 31(23): p. 8476-90.

6. Taylor, S.R., et al., GABAergic and glutamatergic efferents of the mouse ventral tegmental area. Journal of Comparative Neurology, 2014. 522(14): p. 3308-3334.

7. Johnson, S.W. and R.A. North, Opioids excite dopamine neurons by hyperpolarization of local interneurons. J Neurosci, 1992. 12(2): p. 483-8.

8. Jhou, T.C., et al., The rostromedial tegmental nucleus (RMTg), a GABAergic afferent to midbrain dopamine neurons, encodes aversive stimuli and inhibits motor responses. Neuron, 2009. 61(5): p. 786-800.

9. Wilson, C.J., P.M. Groves, and E. Fifkova, Monoaminergic synapses, including dendrodendritic synapses in the rat substantia nigra. Exp Brain Res, 1977. 30(2-3): p. 161-74.

10. Kalivas, P.W. and P. Duffy, A comparison of axonal and somatodendritic dopamine release using in vivo dialysis. J Neurochem, 1991. 56(3): p. 961-7.

11. Lammel, S., et al., Unique properties of mesoprefrontal neurons within a dual mesocorticolimbic dopamine system. Neuron, 2008. 57(5): p. 760-73.

12. Lammel, S., et al., Projection-specific modulation of dopamine neuron synapses by aversive and rewarding stimuli. Neuron, 2011. 70(5): p. 855-62.

13. Lammel, S., B.K. Lim, and R.C. Malenka, Reward and aversion in a heterogeneous midbrain dopamine system. Neuropharmacology, 2014. 76 Pt B: p. 351-9.

14. Lunn, M.L., et al., A unique sorting nexin regulates trafficking of potassium channels via a PDZ domain interaction. Nat Neurosci, 2007. 10(10): p. 1249-59.

15. Kajii, Y., et al., A developmentally regulated and psychostimulant-inducible novel rat gene mrt1 encoding PDZ-PX proteins isolated in the neocortex. Mol Psychiatry, 2003. 8(4): p. 434-44. 
16. Balana, B., et al., Mechanism underlying selective regulation of $G$ protein-gated inwardly rectifying potassium channels by the psychostimulant-sensitive sorting nexin 27. Proc Natl Acad Sci U S A, 2011. 108(14): p. 5831-6.

17. Nakagawa, T. and M. Asahi, beta1-adrenergic receptor recycles via a membranous organelle, recycling endosome, by binding with sorting nexin27. J Membr Biol, 2013. 246(7): p. 571-9.

18. Lauffer, B.E., et al., SNX27 mediates PDZ-directed sorting from endosomes to the plasma membrane. J Cell Biol, 2010. 190(4): p. 565-74.

19. Temkin, P., et al., SNX27 mediates retromer tubule entry and endosome-to-plasma membrane trafficking of signalling receptors. Nat Cell Biol, 2011. 13(6): p. 715-21.

20. Bauch, C., et al., Subcellular sorting of the G-protein coupled mouse somatostatin receptor 5 by a network of PDZ-domain containing proteins. PLoS One, 2014. 9(2): p. e88529.

21. Hussain, N.K., et al., Sorting Nexin 27 regulates basal and activity-dependent trafficking of AMPARs. Proc Natl Acad Sci U S A, 2014. 111(32): p. 11840-5.

22. Loo, L.S., et al., A role for sorting nexin 27 in AMPA receptor trafficking. Nat Commun, 2014. 5: p. 3176.

23. Cai, L., et al., Deficiency of sorting nexin 27 (SNX27) leads to growth retardation and elevated levels of N-methyl-D-aspartate receptor 2C (NR2C). Mol Cell Biol, 2011. 31(8): p. 1734-47.

24. Joubert, L., et al., New sorting nexin (SNX27) and NHERF specifically interact with the 5-HT4a receptor splice variant: roles in receptor targeting. J Cell Sci, 2004. 117(Pt 22): p. 5367-79.

25. Balana, B., et al., Ras-association domain of sorting Nexin 27 is critical for regulating expression of GIRK potassium channels. PLoS One, 2013. 8(3): p. e59800.

26. Ghai, R., et al., Phox homology band 4.1/ezrin/radixin/moesin-like proteins function as molecular scaffolds that interact with cargo receptors and Ras GTPases. Proc Natl Acad Sci U S A, 2011. 108(19): p. 7763-8.

27. Ma, D., et al., Diverse trafficking patterns due to multiple traffic motifs in G proteinactivated inwardly rectifying potassium channels from brain and heart. Neuron, 2002. 33(5): p. 715-29.

28. Munoz, M.B. and P.A. Slesinger, Sorting nexin 27 regulation of $G$ protein-gated inwardly rectifying $K(+)$ channels attenuates in vivo cocaine response. Neuron, 2014. 82(3): $\mathrm{p}$. $659-69$.

29. Steinberg, F., et al., A global analysis of SNX27-retromer assembly and cargo specificity reveals a function in glucose and metal ion transport. Nat Cell Biol, 2013. 15(5): p. 46171. 
30. Matsuda, H., A. Saigusa, and H. Irisawa, Ohmic conductance through the inwardly rectifying K channel and blocking by internal Mg2+. Nature, 1987. 325(7000): p. 156-9.

31. Lopatin, A.N., E.N. Makhina, and C.G. Nichols, Potassium channel block by cytoplasmic polyamines as the mechanism of intrinsic rectification. Nature, 1994. 372(6504): p. 3669.

32. Gahwiler, B.H. and D.A. Brown, GABAB-receptor-activated K+ current in voltageclamped CA3 pyramidal cells in hippocampal cultures. Proc Natl Acad Sci U S A, 1985. 82(5): p. 1558-62.

33. Lacey, M.G., N.B. Mercuri, and R.A. North, On the potassium conductance increase activated by $G A B A B$ and dopamine $D 2$ receptors in rat substantia nigra neurones. $\mathrm{J}$ Physiol, 1988. 401: p. 437-53.

34. North, R.A., et al., Mu and delta receptors belong to a family of receptors that are coupled to potassium channels. Proc Natl Acad Sci U S A, 1987. 84(15): p. 5487-91.

35. Andrade, R. and R.A. Nicoll, Pharmacologically distinct actions of serotonin on single pyramidal neurones of the rat hippocampus recorded in vitro. J Physiol, 1987. 394: p. 99-124.

36. Trussell, L.O. and M.B. Jackson, Adenosine-activated potassium conductance in cultured striatal neurons. Proc Natl Acad Sci U S A, 1985. 82(14): p. 4857-61.

37. Egan, T.M. and R.A. North, Acetylcholine hyperpolarizes central neurones by acting on an M2 muscarinic receptor. Nature, 1986. 319(6052): p. 405-7.

38. Williams, J.T., G. Henderson, and R.A. North, Characterization of alpha 2-adrenoceptors which increase potassium conductance in rat locus coeruleus neurones. Neuroscience, 1985. 14(1): p. 95-101.

39. Logothetis, D.E., et al., The beta gamma subunits of GTP-binding proteins activate the muscarinic K+ channel in heart. Nature, 1987. 325(6102): p. 321-6.

40. Reuveny, E., et al., Activation of the cloned muscarinic potassium channel by G protein beta gamma subunits. Nature, 1994. 370(6485): p. 143-6.

41. Wickman, K.D., et al., Recombinant G-protein beta gamma-subunits activate the muscarinic-gated atrial potassium channel. Nature, 1994. 368(6468): p. 255-7.

42. Dascal, N., et al., Atrial G protein-activated K+ channel: expression cloning and molecular properties. Proc Natl Acad Sci U S A, 1993. 90(21): p. 10235-9.

43. Kubo, Y., et al., Primary structure and functional expression of a rat G-protein-coupled muscarinic potassium channel. Nature, 1993. 364(6440): p. 802-6.

44. Lesage, F., et al., Cloning provides evidence for a family of inward rectifier and G-protein coupled K+ channels in the brain. FEBS Lett, 1994. 353(1): p. 37-42. 
45. Krapivinsky, G., et al., The G-protein-gated atrial K+ channel IKACh is a heteromultimer of two inwardly rectifying $K(+)$-channel proteins. Nature, 1995. 374(6518): p. 135-41.

46. Isomoto, S., et al., A novel ubiquitously distributed isoform of GIRK2 (GIRK2B) enhances GIRK1 expression of the G-protein-gated K+ current in Xenopus oocytes. Biochem Biophys Res Commun, 1996. 218(1): p. 286-91.

47. Lesage, F., et al., Molecular properties of neuronal G-protein-activated inwardly rectifying K+ channels. J Biol Chem, 1995. 270(48): p. 28660-7.

48. Tsaur, M.L., et al., Isolation of a cDNA clone encoding a KATP channel-like protein expressed in insulin-secreting cells, localization of the human gene to chromosome band 21q22.1, and linkage studies with NIDDM. Diabetes, 1995. 44(5): p. 592-6.

49. Kofuji, P., N. Davidson, and H.A. Lester, Evidence that neuronal G-protein-gated inwardly rectifying $K+$ channels are activated by $G$ beta gamma subunits and function as heteromultimers. Proc Natl Acad Sci U S A, 1995. 92(14): p. 6542-6.

50. Lujan, R. and C. Aguado, Localization and Targeting of GIRK Channels in Mammalian Central Neurons. Int Rev Neurobiol, 2015. 123: p. 161-200.

51. Chung, H.J., et al., Neuronal activity regulates phosphorylation-dependent surface delivery of $G$ protein-activated inwardly rectifying potassium channels. Proc Natl Acad Sci U S A, 2009. 106(2): p. 629-34.

52. Karschin, C., et al., IRK(1-3) and GIRK(1-4) inwardly rectifying $K+$ channel mRNAs are differentially expressed in the adult rat brain. J Neurosci, 1996. 16(11): p. 3559-70.

53. Kobayashi, T., et al., Molecular cloning of a mouse G-protein-activated K+ channel (mGIRK1) and distinct distributions of three GIRK (GIRK1, 2 and 3) mRNAs in mouse brain. Biochem Biophys Res Commun, 1995. 208(3): p. 1166-73.

54. Liao, Y.J., Y.N. Jan, and L.Y. Jan, Heteromultimerization of G-protein-gated inwardly rectifying $K+$ channel proteins GIRK1 and GIRK2 and their altered expression in weaver brain. J Neurosci, 1996. 16(22): p. 7137-50.

55. Cruz, H.G., et al., Bi-directional effects of $G A B A(B)$ receptor agonists on the mesolimbic dopamine system. Nat Neurosci, 2004. 7(2): p. 153-9.

56. Inanobe, A., et al., Characterization of G-protein-gated K+ channels composed of Kir3.2 subunits in dopaminergic neurons of the substantia nigra. J Neurosci, 1999. 19(3): p. 1006-17.

57. Jelacic, T.M., et al., Functional and biochemical evidence for G-protein-gated inwardly rectifying $K^{+}($GIRK) channels composed of GIRK2 and GIRK3. J Biol Chem, 2000. 275(46): p. 36211-6.

58. Jelacic, T.M., S.M. Sims, and D.E. Clapham, Functional expression and characterization of G-protein-gated inwardly rectifying K+ channels containing GIRK3. J Membr Biol, 1999. 169(2): p. 123-9. 
59. Labouebe, G., et al., RGS2 modulates coupling between GABAB receptors and GIRK channels in dopamine neurons of the ventral tegmental area. Nat Neurosci, 2007. 10(12): p. 1559-68.

60. Hearing, M., et al., Repeated cocaine weakens $G A B A(B)$-Girk signaling in layer 5/6 pyramidal neurons in the prelimbic cortex. Neuron, 2013. 80(1): p. 159-70.

61. Luscher, C. and R.C. Malenka, Drug-evoked synaptic plasticity in addiction: from molecular changes to circuit remodeling. Neuron, 2011. 69(4): p. 650-63.

62. Chen, R., et al., Abolished cocaine reward in mice with a cocaine-insensitive dopamine transporter. Proc Natl Acad Sci U S A, 2006. 103(24): p. 9333-8.

63. Sulzer, D., et al., Amphetamine redistributes dopamine from synaptic vesicles to the cytosol and promotes reverse transport. J Neurosci, 1995. 15(5 Pt 2): p. 4102-8.

64. Lüscher, C. and M.A. Ungless, The mechanistic classification of addictive drugs, in PLoS Medicine. 2006. p. 2005-2010.

65. Klitenick, M.A., P. DeWitte, and P.W. Kalivas, Regulation of somatodendritic dopamine release in the ventral tegmental area by opioids and GABA: an in vivo microdialysis study. J Neurosci, 1992. 12(7): p. 2623-32.

66. Morgan, A.D., et al., Decreased cocaine self-administration in Kir3 potassium channel subunit knockout mice. Neuropsychopharmacology, 2003. 28(5): p. 932-8.

67. Arora, D., et al., Altered neurotransmission in the mesolimbic reward system of Girk mice. J Neurochem, 2010. 114(5): p. 1487-97.

68. Cruz, H.G., et al., Absence and rescue of morphine withdrawal in GIRK/Kir3 knock-out mice. J Neurosci, 2008. 28(15): p. 4069-77.

69. Kotecki, L., et al., GIRK Channels Modulate Opioid-Induced Motor Activity in a Cell Type- and Subunit-Dependent Manner. J Neurosci, 2015. 35(18): p. 7131-42.

70. Marker, C.L., et al., Spinal G-protein-gated potassium channels contribute in a dosedependent manner to the analgesic effect of mu- and delta- but not kappa-opioids. $\mathrm{J}$ Neurosci, 2005. 25(14): p. 3551-9.

71. Blednov, Y.A., et al., Potassium channels as targets for ethanol: studies of G-proteincoupled inwardly rectifying potassium channel 2 (GIRK2) null mutant mice. J Pharmacol Exp Ther, 2001. 298(2): p. 521-30.

72. Tipps, M.E., et al., G Protein-Gated Inwardly Rectifying Potassium Channel Subunit 3 Knock-Out Mice Show Enhanced Ethanol Reward. Alcohol Clin Exp Res, 2016. 40(4): p. 857-64.

73. Kozell, L.B., et al., Mapping a barbiturate withdrawal locus to a $0.44 \mathrm{Mb}$ interval and analysis of a novel null mutant identify a role for Kcnj9 (GIRK3) in withdrawal from pentobarbital, zolpidem, and ethanol. J Neurosci, 2009. 29(37): p. 11662-73. 
74. Herman, M.A., et al., GIRK3 gates activation of the mesolimbic dopaminergic pathway by ethanol. Proc Natl Acad Sci U S A, 2015. 112(22): p. 7091-6.

75. Lammel, S., et al., Diversity of transgenic mouse models for selective targeting of midbrain dopamine neurons. Neuron, 2015. 85(2): p. 429-38.

76. McCall, N.M., et al., Selective Ablation of GIRK Channels in Dopamine Neurons Alters Behavioral Effects of Cocaine in Mice. Neuropsychopharmacology, 2016.

77. Fujiyama, K., et al., Differential regulation by stimulants of neocortical expression of mrt1, arc, and homer1a mRNA in the rats treated with repeated methamphetamine. Synapse, 2003. 49(3): p. 143-9.

78. Ahmed, B.Y., et al., Efficient delivery of Cre-recombinase to neurons in vivo and stable transduction of neurons using adeno-associated and lentiviral vectors. BMC Neurosci, 2004. 5: p. 4.

79. Huang, C.S., et al., Common molecular pathways mediate long-term potentiation of synaptic excitation and slow synaptic inhibition. Cell, 2005. 123(1): p. 105-18.

80. Kulik, A., et al., Subcellular localization of metabotropic $G A B A(B)$ receptor subunits $G A B A(B 1 a / b)$ and $G A B A(B 2)$ in the rat hippocampus. J Neurosci, 2003. 23(35): p. 11026-35.

81. Drake, C.T., et al., GIRK1 immunoreactivity is present predominantly in dendrites, dendritic spines, and somata in the CA1 region of the hippocampus. Proc Natl Acad Sci U S A, 1997. 94(3): p. 1007-12.

82. Lalive, A.L., et al., Firing modes of dopamine neurons drive bidirectional GIRK channel plasticity. J Neurosci, 2014. 34(15): p. 5107-14.

83. Chung, H.J., et al., G protein-activated inwardly rectifying potassium channels mediate depotentiation of long-term potentiation. Proc Natl Acad Sci U S A, 2009. 106(2): p. 63540.

84. Guetg, N., et al., NMDA receptor-dependent GABAB receptor internalization via CaMKII phosphorylation of serine 867 in GABAB1. Proc Natl Acad Sci U S A, 2010. 107(31): p. 13924-9.

85. Terunuma, M., et al., Prolonged activation of NMDA receptors promotes dephosphorylation and alters postendocytic sorting of GABAB receptors. Proc Natl Acad Sci U S A, 2010. 107(31): p. 13918-23.

86. Beckstead, M.J., et al., Vesicular dopamine release elicits an inhibitory postsynaptic current in midbrain dopamine neurons. Neuron, 2004. 42(6): p. 939-46.

87. Beckstead, M.J. and J.T. Williams, Long-term depression of a dopamine IPSC. J Neurosci, 2007. 27(8): p. 2074-80.

88. Gantz, S.C., et al., Distinct regulation of dopamine D2S and D2L autoreceptor signaling by calcium. Elife, 2015. 4. 
89. Shimosato, K., S. Watanabe, and S. Kitayama, Differential effects of trihexyphenidyl on place preference conditioning and locomotor stimulant activity of cocaine and methamphetamine. Naunyn Schmiedebergs Arch Pharmacol, 2001. 364(1): p. 74-80.

90. Padgett, C.L., et al., Methamphetamine-Evoked Depression of GABA B Receptor Signaling in GABA Neurons of the VTA. Neuron, 2012. 73(5): p. 978-989.

91. Ungless, M.A., et al., Single cocaine exposure in vivo induces long-term potentiation in dopamine neurons. Nature, 2001. 411(6837): p. 583-7.

92. Walker, D.M., et al., Regulation of chromatin states by drugs of abuse. Curr Opin Neurobiol, 2015. 30: p. 112-21.

93. Meye, F.J., et al., Cocaine-evoked negative symptoms require AMPA receptor trafficking in the lateral habenula. Nat Neurosci, 2015. 18(3): p. 376-8.

94. Jhou, T.C., et al., Cocaine drives aversive conditioning via delayed activation of dopamine-responsive habenular and midbrain pathways. J Neurosci, 2013. 33(17): p. 7501-12.

95. Lecca, S., et al., Rescue of GABAB and GIRK function in the lateral habenula by protein phosphatase $2 A$ inhibition ameliorates depression-like phenotypes in mice. Nat Med, 2016. 22(3): p. 254-61.

96. Arora, D., et al., Acute cocaine exposure weakens $G A B A(B)$ receptor-dependent Gprotein-gated inwardly rectifying $K+$ signaling in dopamine neurons of the ventral tegmental area. J Neurosci, 2011. 31(34): p. 12251-7.

97. Munoz, M.B., et al., A Role for the GIRK3 Subunit in Methamphetamine-Induced Attenuation of GABAB Receptor-Activated GIRK Currents in VTA Dopamine Neurons. J Neurosci, 2016. 36(11): p. 3106-14.

98. Kmiotek, E.K., C. Baimel, and K.J. Gill, Methods for intravenous self administration in a mouse model. J Vis Exp, 2012(70): p. e3739.

99. Smith, L.N., et al., Assessment of Cocaine-induced Behavioral Sensitization and Conditioned Place Preference in Mice. J Vis Exp, 2016(108): p. 53107.

100. Sharpe, A.L., et al., Methamphetamine self-administration in mice decreases GIRK channel-mediated currents in midbrain dopamine neurons. Int J Neuropsychopharmacol, 2015. 18(5).

101. Days, E., et al., Discovery and Characterization of a Selective Activator of the G-Protein Activated Inward-Rectifying Potassium (GIRK) Channel, in Probe Reports from the NIH Molecular Libraries Program. 2010: Bethesda (MD).

102. Kaufmann, K., et al., ML297 (VU0456810), the first potent and selective activator of the GIRK potassium channel, displays antiepileptic properties in mice. ACS Chem Neurosci, 2013. 4(9): p. 1278-86. 


\section{Figure Legends:}

Figure 1: (a) Current-voltage plots for GIRK channels (red) and voltage-gated $\mathrm{K}^{+}$channels $\left(\mathrm{K}_{\mathrm{v}}\right.$, orange). Like all inward rectifiers $\left(\mathrm{K}_{\mathrm{IR}}\right)$, GIRK channels conduct a small outward $\mathrm{K}^{+}$current at the resting membrane potential $\left(\mathrm{V}_{\text {rest }}\right)$ and a large inward current at potentials more negative than the equilibrium potential, i.e., reversal potential, for potassium $\left(E_{K}\right)$. $K_{V}$ channels conduct a large outward current at depolarized membrane potentials. (b) There are three primary GIRK channel subunits in the brain that form hetero- and homotetrameric channels. Notably, GIRK1-GIRK2 heterotetramers are most common in the brain, while GIRK2 is the only subunit that can form functional homotetramers. (c) GIRK channel subunits contain different motifs in the N- and Cterminal domains that affect trafficking.

Figure 2: Neuronal subtypes and projections within the brain's reward system where exposure to psychostimulants or stress produces decreases in GABA $_{B}$-GIRK currents (I Baclofen$_{\text {). }}$. Glutamatergic neurons (green) in the mPFC and LHb $[60,95]$, and GABAergic neurons (red) in the VTA [90], undergo downregulation of baclofen-activated GIRK currents ( $I_{\text {baclofen }}$ ) via a PP2A/GABA ${ }_{\mathrm{B} 2}$ dephosphorylation mechanism (red arrow). By contrast, psychostimulants also induce a decrease in $I_{\text {Baclofen }}$ in $I_{h}$-positive VTA DA neurons (blue), but the mechanism involves the GIRK3 subunit and not PP2A/GABA ${ }_{B 2}$ dephosphorylation (blue arrow) [28]. Selfadministration of methamphetamine also causes down-regulation of $I_{\text {Baclofen }}$ in SN DA neurons, but the mechanism is less well understood [100]. Currently, there are no studies investigating the effect of psychostimulants on SN GABA neurons or on $I_{h}$-negative $\left(I_{h}{ }^{-}\right)$VTA DA neurons that project to mPFC or amygdala (not shown). Note that not all neuronal projections are shown for clarity. 


\section{Outstanding Questions}

1. In which cell types in the brain does loss of GIRK channels lead to altered drug-related behaviors?

2. Are GIRK channels subject to unique regulation in subpopulations of VTA DA neurons that project to different target regions in the mesocorticolimbic system?

3. What is the role of GIRK channels in addiction in brain regions (e.g. lateral habenula, hippocampus) outside the mesocorticolimbic DA system?

4. What are all of the molecular steps by which exposure to psychostimulants causes a persistent downregulation of $\mathrm{GABA}_{B} \mathrm{R}-\mathrm{GIRK}$ currents?

5. Can small molecule modulators of specific GIRK channels be used to treat addiction? 
Figure 1

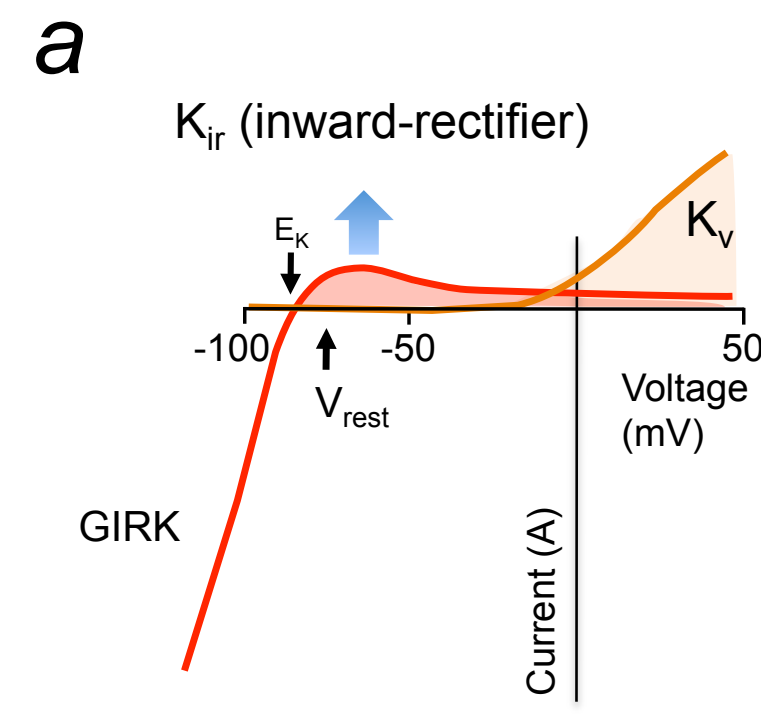

$b$

Heterotetramer

Homotetramer

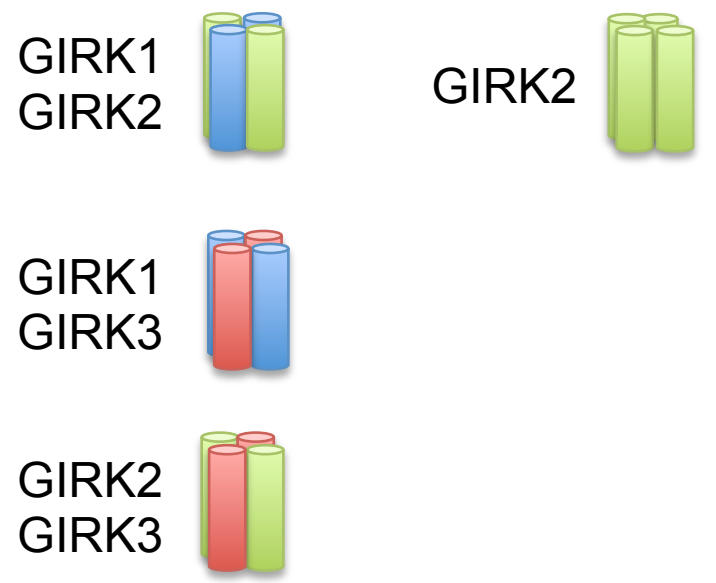

C
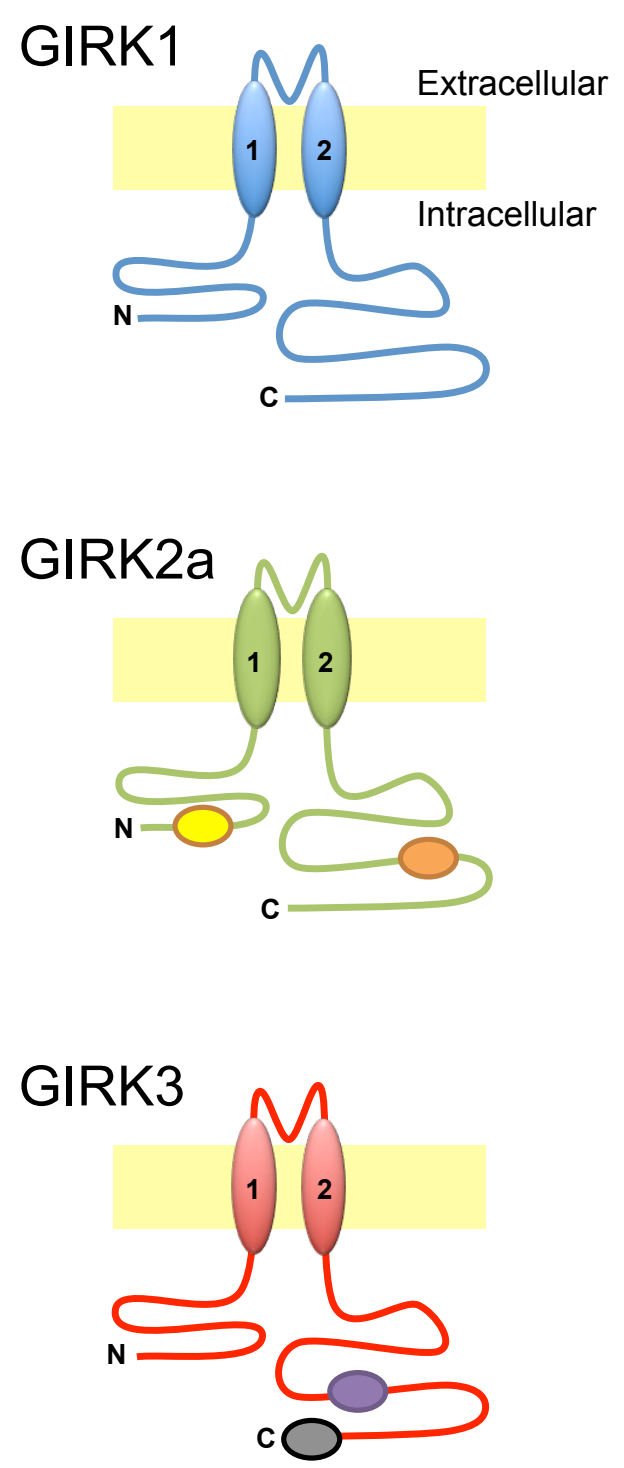

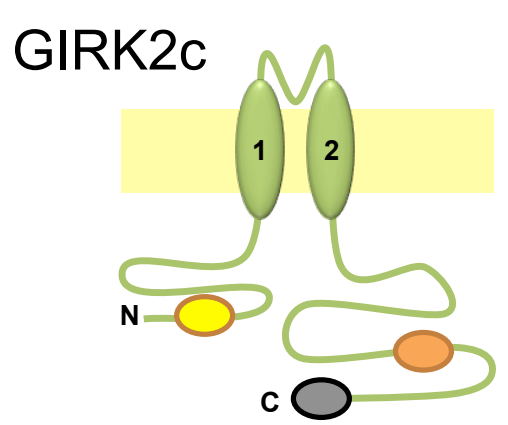

Motifs:

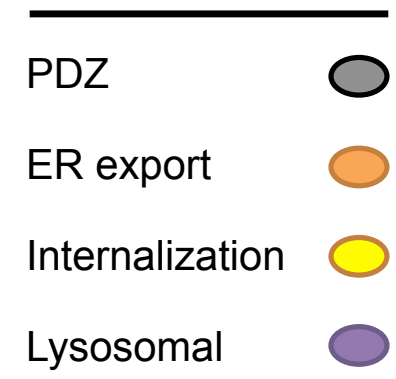




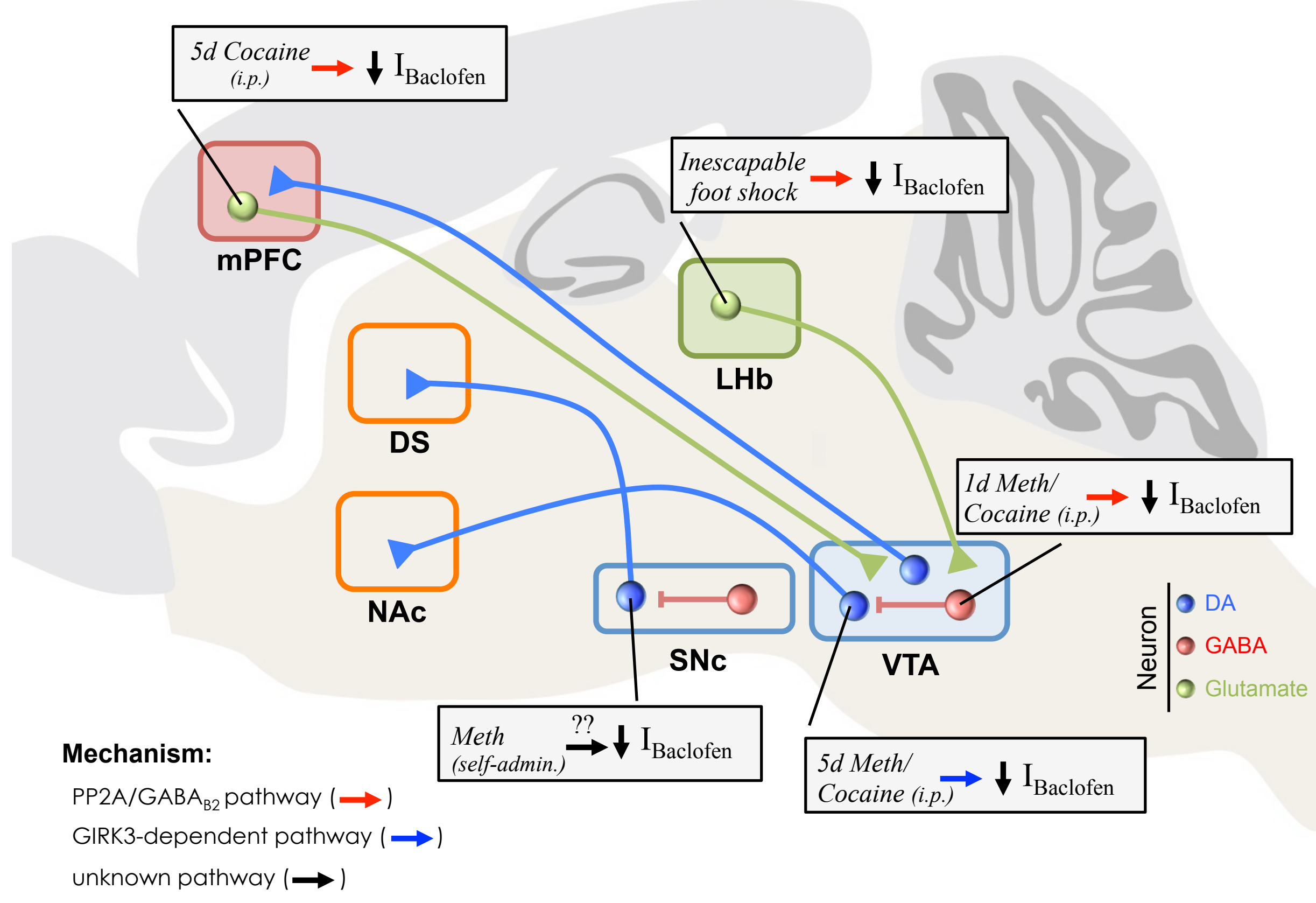


Table 1: Effects of GIRK and related knockouts on drug-related behaviors

\begin{tabular}{|c|c|c|c|c|c|c|}
\hline Gene & Cell type & Drug & \multicolumn{2}{|c|}{ Behavior } & lGIRK & Citation \\
\hline \multirow{2}{*}{$\begin{array}{l}\text { Girk1 } \\
\text { (Kcnj3) }\end{array}$} & \multirow[t]{2}{*}{ Whole-animal } & Cocaine & $\uparrow$ & Locomotor activity & nc IBaclofen VTA DA & Arora et al., 2010; Labouébe et al., 2007 \\
\hline & & DAMGO & nc & Locomotor activity & $\downarrow \downarrow$ IDAMGO VTA GABA & Kotecki et al., 2015 \\
\hline \multirow{6}{*}{$\begin{array}{l}\text { Girk2 } \\
\text { (Kcnj6) }\end{array}$} & \multirow[t]{6}{*}{ Whole-animal } & Cocaine & $\uparrow \uparrow$ & Locomotor activity & $\downarrow \downarrow \quad$ IBaclofen VTA DA & Arora et al., 2010; Morgan et al., 2003 \\
\hline & & Cocaine & $\downarrow \downarrow$ & Self-administration & - & Morgan et al., 2003 \\
\hline & & - & & & $\downarrow \downarrow$ IBaclofen VTA DA \& GABA & Cruz et al., 2004; Labouébe et al., 2007 \\
\hline & & Morphine & nc & Locomotor activity & $\downarrow \downarrow$ IBaclofen VTA GABA & Kotecki et al., 2015 \\
\hline & & Ethanol & $\uparrow$ & Consumption & - & Blednov et al., 2001 \\
\hline & & Morphine & $\uparrow$ & Withdrawal & $\downarrow \quad I_{\text {Morphine LC }}$ & Cruz et al., 2008 \\
\hline \multirow{8}{*}{$\begin{array}{l}\text { Girk3 } \\
\text { (Kcnj9) }\end{array}$} & \multirow[t]{8}{*}{ Whole-animal } & Cocaine & $\mathrm{nc}$ & Locomotor activity & nc IBaclofen VTA DA & Morgan et al., 2003; Arora et al., 2010 \\
\hline & & Cocaine & $\downarrow \downarrow$ & Self-administration & - & Morgan et al., 2003 \\
\hline & & - & & & $\downarrow /$ nc IBaclofen VTA DA & Labouébe et al., 2007; Arora et al., 2010 \\
\hline & & Morphine & $\downarrow$ & Locomotor activity & nc I I Baclofen VTA GABA & Kotecki et al., 2015 \\
\hline & & Ethanol & $\downarrow$ & Sedative-hypnotic withdrawal & - & Kozell et al., 2009 \\
\hline & & Ethanol & $\uparrow$ & Binge drinking & - & Herman et al., 2015 \\
\hline & & Ethanol & $\uparrow$ & CPP & - & Tipps et al., 2016 \\
\hline & & Morphine & nc & Withdrawal & $\downarrow \downarrow$ IMorphine LC & Cruz et al., 2008 \\
\hline \multirow[t]{4}{*}{ Girk2/3 } & \multirow[t]{4}{*}{ Whole-animal } & Cocaine & nc & Locomotor activity & - & Morgan et al., 2003 \\
\hline & & Cocaine & $\downarrow$ & Self-administration & - & Morgan et al., 2003 \\
\hline & & - & & & $\downarrow \downarrow$ IBaclofen VTA DA & Labouébe et al., 2007 \\
\hline & & Morphine & $\downarrow \downarrow$ & Withdrawal & $\downarrow \quad I_{\text {Morphine LC }}$ & Cruz et al., 2008 \\
\hline \multirow[t]{4}{*}{ Girk2 } & \multirow[t]{4}{*}{ DA neurons } & Morphine & nc & Locomotor activity & $\downarrow \downarrow$ IBaclofen VTA DA & Kotecki et al., 2015 \\
\hline & & Cocaine & $\uparrow$ & Locomotor activity & $\downarrow \quad$ lQuinpirole VTA DA & McCall et al., 2016 \\
\hline & & Cocaine & $\uparrow$ & Locomotor sensitization & $\downarrow \quad$ lQuinpirole VTA DA & McCall et al., 2016 \\
\hline & & Cocaine & $\uparrow$ & Self-administration & $\downarrow \quad$ lQuinpirole VTA DA & McCall et al., 2016 \\
\hline Girk2 & GABA neurons & Morphine & $\mathrm{nc}$ & Locomotor activity & $\downarrow \quad$ IBaclofen VTA GABA & Kotecki et al., 2015 \\
\hline Snx27 & DA neurons & Cocaine & $\uparrow \uparrow$ & Locomotor activity & $\downarrow \quad I_{\text {Baclofen VTA DA }}$ & Munoz and Slesinger, 2014 \\
\hline
\end{tabular}

Legend: $\downarrow$ - intermediate effect; $\downarrow \downarrow$ - maximal effect; '-': not tested; nc: no change. 